\title{
Embryological and clinical implications of the association between anorectal malformations and spinal dysraphisms
}

\author{
Giorgia Totonelli ${ }^{1}$ - Raffaella Messina ${ }^{2}$ Francesco Morini ${ }^{1} \cdot$ Giovanni Mosiello $^{3}$ • \\ Paolo Palma $^{2} \cdot$ Marianna Scuglia $^{1} \cdot$ Barbara D. Iacobelli $^{1} \cdot$ Pietro Bagolan $^{1}$
}

Accepted: 30 May 2017

(C) Springer-Verlag GmbH Germany 2017

\begin{abstract}
Purpose To describe the association of anorectal malformation (ARM) and spinal dysraphism (SD) in terms of impact on the management of SD and embryogenetic implications.

Methods Patients with SD associated with (A) or without (B) ARM were included. The two groups were further divided into operated on (A1/B1) or not (A2/B2) for SD. Groups A and B were compared for type of SD (embryogenetic classification) and prevalence of neurosurgery; Groups A1 vs. A2 for type of ARM (Wingspread classification); Groups A1 vs. B1 for age at neurosurgery, neurophysiology, and clinical symptoms.

Main results One hundred twenty-one patients with SD, 83 with and 38 without ARM were consecutively treated (1999-2015). Group A was associated only with SDs developing after primary neurulation, corresponding to the period of cloacal septation and organogenesis $(p=0.0007)$. Untethering surgery was significantly less frequent in Group A $(p<0.0001$ and $p=0.04$, respectively). Higher ARMs were not associated with increased risk for neurosurgery. No other significant differences were detected.
\end{abstract}

Giorgia Totonelli

gtotonelli@gmail.com

1 Neonatal Surgery Unit, Department of Medical and Surgical Neonatology, Bambino Gesù Children's Hospital, IRCCS, Piazza S. Onofrio, 4, 00165 Rome, Italy

2 Neurosurgery Unit, Department of Neuroscience and Neurorehabilitation, Bambino Gesù Children's Hospital, IRCCS, Rome, Italy

3 Neuro-Urology Unit, Department of Surgery, Bambino Gesù Children's Hospital, IRCCS, Rome, Italy
Conclusions In our series, ARMs were associated only with SD developing after primary neurulation, suggesting a single insult leading to both SD and the associated ARM. Neurosurgery is indicated less frequently in patients with ARM-associated SD, despite the similar preoperative clinical features.

Keywords Anorectal malformations - Spinal dysraphism . Tethered cord · Spinal lipoma $\cdot$ Embryogenesis .

Neurulation

\section{Introduction}

The association of anorectal malformation (ARM) with other congenital anomalies has been widely described in the literature $[1,2]$. Among those, the presence of spinal dysraphism (SD) has been reported in up to $52 \%$ of patients with ARMs [3]. This frequent coexistence suggests a possible developmental association. However, few studies have analyzed the potential developmental association of $\mathrm{ARM}$ and SD.

From a clinical point of view, SD may lead to the tethered cord syndrome (TCS), which refers to the abnormal fixation of the spinal cord to a structure that limits its movements in the craniocaudal directions. In patients with SD associated with ARMs, the TCS symptoms may sometimes overlap clinical manifestations of the ARM and sequelae of its surgical correction (i.e., lower urinary tract and fecal dysfunctions). This potential overlap still leads to controversies on neurosurgical indications of SD, specifically TCS, associated with ARM [3].

In the present study, we aimed to address the following questions: 


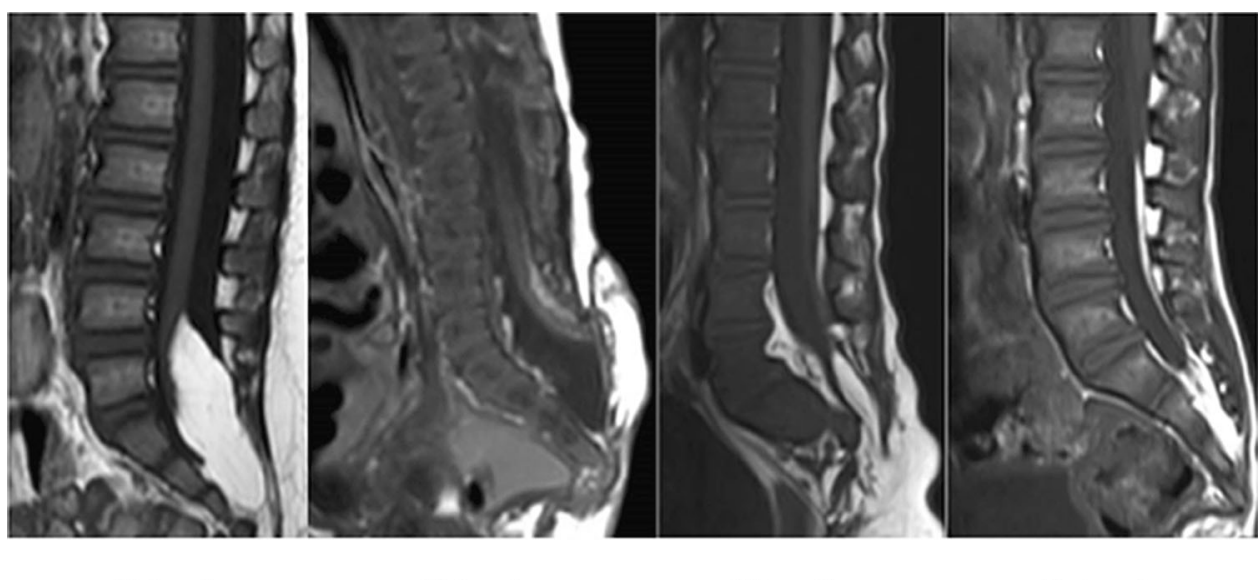

SL. type 1

SL. type 2

Fig. 1 Sagittal T1-weighted MR images of SL in our series in accordance with the Morota's classification system based on embryonic stage. Type $1 \mathrm{SL}$ : the lipoma has a predominant dorsal location, compressing the spinal cord ventrally but preserving the cone shape. Type 2 SL: the end of the spinal cord merges with the lipoma and there is no clear borderline; the conus medullaris is not formed and

(1) Does a correlation exist between ARM and a specific SD type? (2) Can an embryonic relationship be postulated for the frequent coexistence of ARM and SD? (3) Do associated ARMs have an impact on the management of patients with SD?

\section{Materials and methods}

A retrospective study was performed on patients with SD associated with ARM (Group A) and with isolated SD (Group B). Group A included all patients treated for ARMs in the neonatal period at our Institution between January 1999 and December 2015, with associated SD. SD was suspected at spinal US and confirmed at spinal MRI at 1 year of age [4]. Patients diagnosed with isolated SD and referred to the Neurosurgery Unit in the same period were included in Group B. Tethered spinal cord refers to MRI images of a conus medullaris below the L2-L3 vertebra level, associated or not with SD. Tethered spinal cord can develop in symptomatic TCS, with patient presenting with neuro-motor disorders of the back and legs, urinary, and/or bowel dysfunction. In case of syrinx at MRI in asymptomatic patients, they underwent a neuroimaging follow-up in order to control the progression of the syringomyelia. Patients were further divided into operated on (Group A1/ B1) or not (Group A2/B2) for SD. Indication to neurosurgery was given in patients with symptomatic and/or worsening TCS, when the MRI showed progression of the syringomyelia, or in SDs at high risk of developing TCS. ARMs were classified according to Wingspread classification [5]. SDs were classified in spinal lipomas (SL) the neural tissue extrudes from the spinal canal. Type 3 SL: the lipoma is caudal to the conus medullaris and there is no fascial or dural defect; the lipoma passes through the sacral hiatus merging with the subcutaneous fat. Type 4 SL: filum lipoma is a terminal lipoma; normal conus medullaris

following the "New classification of spinal lipomas based on embryonic stage" [6] (Fig. 1) and other SDs according to the clinical classification of spinal neural tube defects [7], including abnormal filum terminale, dermal sinus, and hydromyelia. As known, SLs type 1 and 2 arise during primary neurulation; SLs type 3 and 4 develop after primary neurulation; the other types of SD observed in the study group derive from a secondary neurulation failure.

Groups A and B were compared for the type of SD, based on the embryonic stage of development. Groups A and $\mathrm{B}$ were also compared for prevalence of neurosurgery. Groups A1 and B1 were compared for age at neurosurgery, preoperative neurophysiological data, preoperative bladder dysfunction, clinical symptoms, and neurologic examination, before and after neurosurgery, in terms of neurologic improvement. All patients underwent urodynamic studies after 2 years of age. Urodynamic studies allow evaluating overactivity or underactivity of detrusor, filling pressure, pressure-flow relationship between the bladder and the urethra through the simultaneous measurement of bladder, abdominal pressure, and subtracted detrusor pressure, in order to diagnose a lower urinary tract dysfunction and its pathophysiology. Preoperative neurophysiological studies consisted of Somatosensory Evoked Potentials-SEP, Motor Evoked Potentials-MEP, and Bulbocavernosus Reflex-BCR. In all the operated patients, surgery was assisted by nerve neurophysiologic monitoring and mapping. Neurosurgical approach depended on the type of SD. In SL type 4, surgery consisted of laminotomy (often on two levels, L4-L5) and excision of the lipoma (fatty filum terminale). A reconstructive surgery was performed in the other types of SD, when the malformation consisted of a 
Table 1 SD in patients with (group A) and without (group B) ARM: classification and prevalence based on embryonic phase

\begin{tabular}{lllll}
\hline & Embryonic phase & $\begin{array}{l}\text { Group A } \\
n=83\end{array}$ & $\begin{array}{l}\text { Group B } \\
n=38\end{array}$ & $p$ \\
\hline Type 1 SL $[n(\%)]$ & Primary neurulation failure & 0 & $1(2.6 \%)$ & 0.0007 \\
Type 2 SL $[n(\%)]$ & Junctional neurulation failure & 0 & $5(13.1 \%)$ & \\
Type 3 SL $[n(\%)]$ & Secondary neurulation failure (early phase) & $2(2.4 \%)$ & $10(26.3 \%)$ & \\
Type 4 SL $[n(\%)]$ & Secondary neurulation failure (late phase) & $57(68.7 \%)$ & $13(34.2 \%)$ & \\
Other SD $[n(\%)]$ & Secondary neurulation failure & $24(28.9 \%)$ & $9(23.7 \%)$ & \\
\hline
\end{tabular}

Table 2 Patients characteristics

\begin{tabular}{llll}
\hline & $\begin{array}{l}\text { Group A } \\
n=83\end{array}$ & $\begin{array}{l}\text { Group B } \\
n=38\end{array}$ & $p$ \\
\hline Neurosurgery $[n(\%)]$ & $10(12 \%)$ & $24(63 \%)$ & $<0.0001$ \\
\hline & $\begin{array}{l}\text { Group A1 } \\
n=10\end{array}$ & $\begin{array}{l}\text { Group B1 } \\
n=24\end{array}$ & $p$ \\
\hline $\begin{array}{l}\text { Mean age at neurosurgery (mths) } \\
\begin{array}{l}\text { Pre-op abnormal } \\
\text { neurophysiology }[n(\%)]\end{array}\end{array}$ & $\begin{array}{l}60.1 \\
\begin{array}{l}\text { Pre-op bladder } \\
\text { dysfunction }[n(\%)]\end{array}\end{array}$ & 52.3 & 0.04 \\
\begin{tabular}{l} 
Post-op improvement $[n(\%)]$ \\
\hline
\end{tabular} & $10(100 \%)$ & $14(58 \%)$ & $\mathrm{ns}$ \\
\hline
\end{tabular}

fascial and dural defect through which the lipoma, tethered to the conus medullaris, herniated out of the spinal cord.

\section{Statistical analysis}

Data were analyzed using GraphPad Prism 5.0 Macintosh Version (GraphPad Software, San Diego CA USA, http:// www.graphpad.com). Groups were compared using the Fisher's exact test and $\chi^{2}$ test for trend as appropriate. Results are reported as mean and percentage; $p \geq 0.05$ (two-sided) was considered statistically significant.

\section{Results}

Main results are shown in Tables 1 and 2. During the study period, 83 patients ( 36 females and 47 males) were diagnosed with ARM-associated SD (group A) and 38 patients (19 females and 19 males) with isolated SD (group B). Table 1 shows the distribution of different types of SD in both groups. In our series, the distribution of different types of SD was significantly different between the two groups (Table $1 ; p=0.0007)$. In particular, Group A was associated only with SD developing after the primary neurulation as compared to Group B that showed 16\% SD developing before the secondary neurulation phase (Table $1 ; p<0.0001$ ).
Untethering surgery was significantly less frequent $(p<0.0001)$ and performed at a significantly higher mean age $(p=0.04)$ in ARM-associated SD (Table 2).

All Group A1 patients were symptomatic for TCS at the time of surgery, with abnormal reflexes or gait disturbances, weakness or pain in the legs or other neuro-motor deficits at the neurological examination. Conversely, six patients $(25 \%)$ in group B with type 1/2 SL underwent prophylactic surgery, in order to prevent the development of a TCS, strictly related to the complexity of their spinal dysraphism.

Urodynamic studies showed a bladder dysfunction in all patients preoperatively investigated (80\% in the group A1 and $67 \%$ in the group B1), defined by the lack of synergy between the detrusor and sphincter activity.

No significant difference was observed between the two operated groups in terms of preoperative neurophysiological study and bladder dysfunction (Table 2).

There were no complications related to neurosurgery. We recorded a good outcome and a postoperative improvement, in terms of pain, fatigue, or reflex abnormality resolution in all operated patients in both groups.

\section{Discussion}

The present study showed two major findings. First, patients with ARM-associated SD had a recognizable pattern of type of dysraphism. Second, patients with ARMassociated SD underwent neurosurgery less frequently and at an older age, as compared to those with isolated SD.

SDs include several types of spinal developmental malformations. The spinal cord develops following either the primary or secondary neurulations. Failed neurulation leads to SD, whose type depends on the stage of the failure. The classification of SD used in this study is based on the stages of neural tube formation during embryogenesis, and includes four types of SL and other anomalies as abnormal filum terminale, dermal sinus, and hydromyelia. SLs are among the most common spinal developmental malformations encountered in the pediatric population. They derive from a premature disjunction between the neural and cutaneous ectoderm during the process of primary neurulation. This allows the invasion of mesenchymal tissue into 
the neural structure, leading to the formation of a SL [6]. Failure of primary neurulation results in Type $1 \mathrm{SL}$; type 2 derives from a failure between the primary and the secondary stages (junctional neurulation phase); Type 3 develops after a failure in the early phase secondary neurulation, while Type 4 results from a failure in the late phase secondary neurulation. The other SDs considered (including abnormal filum terminale, dermal sinus, hydromyelia) develop during the secondary neurulation [6]. In our series, ARMs were only associated with only type 3 and $4 \mathrm{SL}$ and with other SDs, all developing after the primary neurulation, as compared to patients with isolated SD $(p<0.0001)$. The anorectal and urogenital organs develop with the cloacal septation, starting after the postovulatory week 5 and ending at postovulatory week 8 , and failure in the cloacal septation results in ARM [8]. This period overlaps with the secondary neurulation, in our series; the frequent association of ARM with specific SD types suggests that a common embryogenic insult occurring between the 5th and 8th postovulatory week may lead to both the SD and the ARM.

Neurosurgical management of ARM-associated SD remains controversial, with some suggesting primary neurosurgery while others proposing a "wait and see" approach $[1,9,10]$. This is mainly due to the potential overlap between the ARM-related clinical manifestations and those due to the associated SD. In fact, SD can present with a wide spectrum of clinical manifestations, varying from an asymptomatic patient to a severe neurological condition, including, but not limited to, sensory-motor abnormalities, the TCS. The sequelae of the ARM surgical correction (i.e., lower urinary tract dysfunctions and fecal incontinence) may mimic manifestations of SD. Hence, in patients with ARMs, early symptoms of SD are sometimes difficult to recognize. In the present study, patients with SD associated with ARM underwent neurosurgery significantly less frequently than patients with isolated SD. These findings may depend on the difficulty to discriminate between ARM-related clinical manifestations from SD-related ones. Also, some patients with isolated SD were operated upon prophylactically. These were patients with type 1 and 2 SL, that have a tight relationship with the conus medullaris, usually with a predominant dorsal location to the conus, and are associated to a higher risk to develop a symptomatic TCS. Therefore, the difference in type of lipoma may be responsible for the difference in the proportion of patients who underwent neurosurgery we found between the two groups. The fact that all patients with ARM and SD who underwent untethering surgery had improvement of clinical sensory-motor signs suggests that their clinical manifestations were not only due to the associated ARM or its treatment, otherwise they would not have experienced clinical improvements after neurosurgery, but were likely due to SD itself. Our findings suggest that the association of ARM and SD may require the delay of neurosurgery. Therefore, it is crucial to perform serial objective clinical and instrumental evaluations, (i.e., MRI, urodynamic studies; SEP, MEP, BCR depending also on the severity of the symptoms referred), to better define the origin of the symptoms reported by the affected patients. This is particularly true during periods of toilet training (3-5 years of age), when clinical manifestations may become apparent and period of rapid growth (6-12 years of age), when the elongation of the body may cause the stretching of a tethered cord and thereby lead to the onset of the symptoms of TCS.

Despite patients with high type ARM seem to have a higher risk of associated SD [11], we did not find a significant difference in neurosurgery prevalence between high, intermediate, and low ARMs in the present study. Since, the type of ARM seems not to predict the risk to develop a TCS requiring surgery, the screening path (i.e., spinal US, MRI) of all patients with ARM is crucial, since even low type ARM may be associated with symptomatic $\mathrm{SD}$ requiring neurosurgery.

\section{Conclusions}

Our study has some limitations mainly due to its retrospective nature and to the limited number of patients with isolated SD. Despite these limitations, the comparison of the two study-groups treated in the same pediatric Institution by a multidisciplinary team highlights the real possibility that the coexistence of ARM and SD could mislead the indication to neurosurgery. Diagnostic tests (MRI, SEP, MEP, BCR, urodynamic studies) should be performed in all ARM patients with associated SD to guide more objectively the management of these complex patients. This in turn will prevent from underestimating symptoms to be related to the tethered spinal cord avoiding the delay of its neurosurgical treatment. Our data finally suggest that ARMs are associated with a specific type of SD, possibly developing during the same embryonic period, between the 5th and 8th week of gestation that is the same period of the secondary neurulation. A single embryonic insult or failure may be responsible for both anomalies. However, further experimental studies are needed to confirm our speculation.

\section{References}

1. Uchida K, Inoue M, Matsubara T, Otake K, Koike Y, Okugawa Y et al (2007) Evaluation and treatment for spinal cord tethering in patients with anorectal malformations. Eur J Pediatr Surg 17(6):408-411 
2. Totonelli G, Catania VD, Morini F, Fusaro F, Mosiello G, Iacobelli BD, Bagolan P (2015) VACTERL association in anorectal malformation: effect on the outcome. Pediatr Surg Int 31(9):805-808. doi:10.1007/s00383-015-3745-5

3. Van den Hondel D, Sloots C, de Jong TH, Lequin M, Wijnen R (2016) Screening and treatment of tethered spinal cord in anorectal malformation patients. Eur J Pediatr Surg 26(1):22-28. doi: $10.1055 / \mathrm{s}-0035-1563673$

4. Scottoni F, Iacobelli BD, Zaccara AM, Totonelli G, Schingo AM, Bagolan P (2014) Spinal ultrasound in patients with anorectal malformations: is this the end of an era? Pediatr Surg Int 30(8):829-831. doi:10.1007/s00383-014-3546-2

5. Stephens FD, Durham-Smith E (1986) Classification, identification, and assessment of surgical treatment of anorectal anomalies. Pediatr Surg Int 1:200-205

6. Morota N, Ihara S, Ogiwara H (2017) New classification of spinal lipomas based on embryonic stage. J Neurosurg Pediatr 19(4):428-439
7. McComb JC (2015) A practical clinical classification of spinal neural tube defects. Childs Nerv Syst 31(10):1641-1657. doi:10. 1007/s00381-015-2845-9

8. Kluth D (2010) Embryology of anorectal malformations. Semin Pediatr Surg 19(3):201-208. doi:10.1053/j.sempedsurg.2010.03. 005 (review)

9. Tuuha SE, Aziz D, Drake J, Wales P, Kim PC (2004) Is surgery necessary for asymptomatic tethered cord in anorectal malformation patients? J Pediatr Surg 39(5):773-777

10. Kyrklund K, Pakarinen MP, Taskinen S, Kivisaari R, Rintala RJ (2016) Spinal cord anomalies in patients with anorectal malformations without severe sacral abnormalities or meningomyelocele: outcomes after expectant, conservative management. J Neurosurg Spine 25(6):782-789

11. Totonelli G, Morini F, Catania VD, Schingo PM, Mosiello G, Palma P, Iacobelli BD, Bagolan P (2016) Anorectal malformations associated spinal cord anomalies. Pediatr Surg Int 32(8):729-735 